\title{
Meaningful Use and Electronic Laboratory Reporting: Challenges Health Information Technology Vendors Face in Kentucky
}

\author{
Michael Abisa, MHI, MBI, MPH \\ University of Kentucky, Lexington, Kentucky, USA
}

\begin{abstract}
:
Objectives: To explore the challenges Health Information Technology (HIT) vendors face to satisfy the requirements for Meaningful Use (MU) and Electronic Laboratory Reporting (ELR) of reportable diseases to the public health departments in Kentucky.

Methodology: A survey was conducted of Health Information Exchange (HIE) vendors in Kentucky through the Kentucky Health Information Exchange (KHIE). The survey was cross-sectional. Data were collected between February and March 2014. Participants were recruited from KHIE vendors. Participants received online survey link and by email and asked to submit their responses. Vendors' feedback were summarized and analyzed to identify their challenges. Out of the 55 vendors who received the survey, 35(63.64\%) responded.
\end{abstract}

Results: Of the seven transport protocol options for ELR, vendors selected virtual private network (VPN) as the most difficult to implement (31.7\%). Secure File Transfer Protocol (SFTP) was selected as preferred ELR transport protocol (31.4\%). Most of the respondents, $80 \%$ responded that they do not have any challenge with the Health Level 7 (HL7) standard implementation guide required by MU for 2014 ELR certification.

Conclusion: The study found that the most difficult transport protocol to implement for ELR is VPN and if vendors have preference, they would use SFTP for ELR over KHIE choice of VPN and Simple Object Access Protocol (SOAP). KHIE vendors do not see any variability in what is reportable by different jurisdiction and also it is not difficult for them to detect what is reportable from one jurisdiction verse the other.

Keywords: Meaningful Use; Electronic Laboratory Reporting; Health Information Exchange; Health Level 7; Health Information Technology; Transport Protocol

Correspondence: Michael Abisa, University of Kentucky, 513-828-9165, mab224@uky.edu, michaelabisa@gmail.com

DOI: 10.5210/ojphi.v9i3.7491 copy articles without permission of the copyright owner(s), as long as the author and OJPHI are acknowledged in the copy and the copy is used for educational, not-for-profit purposes. 


\section{Introduction}

Public Health Departments or agencies rely on hospitals, clinics, and laboratory data to provide timely intervention in a community when there is an outbreak of disease to prevent additional illness. Because the conventional methods of reporting via mail, facsimile, or telephone require active participation of laboratory staff, automated reporting from clinical laboratories has been proposed as a means to improve the quality and timeliness of disease notification [1].

To this end, Electronic Laboratory Reporting (ELR) has been promoted as being integral to improve disease surveillance [2]. At its simplest, Electronic Laboratory Reporting is the distribution of the results of laboratory testing using electronic transmission systems rather than paper-or-fax based processes [3]. Electronic Laboratory Reports are critical for an effective public health response both for routinely reportable diseases as well as potential bioterrorism (BT) agents [4]. With respect to public health disease surveillance activities, ELR is useful for conditions where the diagnosis can be based solely on positive (or negative) results from laboratory testing, such as Chlamydia and Salmonella infection, among others [3]. It is important to mention here that ELR is also useful for conditions that require clinical diagnosis as well. The "usefulness" of ELR is largely driven by public health investigation and reporting. Over the past decades, public health institutions have made much progress in creating secure systems for electronic data transmission to improve the quality of laboratory reporting.

Public health agencies require health information technology vendors to provide secure transport protocols capable of meeting the requirements of Meaningful Use (MU) and ELR. Meaningful use is using certified electronic health record (EHR) technology to:

- Improve quality, safety, efficiency and reduce health disparities

- Engage patients and family

- Improve care coordination, and population and public health

- Maintain privacy and security of patient health information [5].

Interestingly, these agencies do not know whether vendors encounter any challenges in meeting the requirements of meaningful use.

To improve ELR, it is important to understand challenges vendors go through, if any, in meeting the requirements for MU. Identifying these barriers could create an appropriate dialogue between stakeholders to find ways that can improve the system.

To accelerate adoption of ELR, the Center of Disease Control and Prevention (CDC) advanced standards for vocabulary, format, and messaging; funded the development of software; and conducted an extensive outreach campaign to state and local health departments to increase use of the software [6]. To this end, Kentucky state and local public health departments have adopted electronic laboratory reporting for reportable diseases to enable hospitals, clinics, and laboratories to submit lab reports electronically. Clinical laboratory reporting (as opposed to reporting by health care providers) has become increasingly valuable in disease surveillance $[7,8]$. Major public health threats in recent years in the United States support the call for public health data sources integration which will yield to the benefits of all stakeholders involve. 
Currently, Health Information Exchange (HIE) and Public Health Departments across the country have been working with Health Information Technology (HIT) vendors to improve ELR for public health. Improved reporting can help public health departments better allocate limited resources for targeted investigations and interventions [9].

Health Information Exchange provides the infrastructure for information exchange, including the business model, governance structure, operating principles, legal model, and technology model for the exchange of healthcare information among various organizations [10]. Kentucky Health Information Exchange (KHIE) in collaboration with providers and public health departments in Kentucky are working to help state vendors to meet the requirements of ELR as envisaged by the CDC. KHIE acts as a hub between public health departments and providers in transmitting laboratory reports for reportable diseases. The role of Health Information Exchange in providing services to providers and health departments can be seen in three scenarios as provided by the office of the national coordinator.

Scenario 1: Complete Electronic Health Records

Scenario 2: Electronic Health Records and/or Laboratory Information Systems (MU complaint messages)

Scenario 3: Electronic Health Records and/or Laboratory Information Systems [11]. In the first and second scenarios, a provider uses fully or modularly certified technology to report directly to the public health department or through and HIE. Scenario one and two do not require any manipulation of message or content on the part of the HIE. The HIE primary "brokers" the exchange. However, in the third scenario, the provider is not using certified technology and therefore uses the HIE as an extension of their technology to attest to meaningful use.

There are various types of transport protocols vendors' use in transmitting laboratory reports to the public health departments.

A panel of subject matter experts (SME) established by CDC in 2011 evaluated and analyzed five transport protocol options (i.e. Public Health Information Network Messaging System (PHINMS), Simple Mail Transfer Protocol and Multipurpose Internet Mail Extensions (SMTP+S/MIME), Secure File Transfer Protocol (SFTP), Hypertext Transfer Protocol Secure Representational State Transfer (HTTPS REST) and Simple Object Access Protocol (SOAP) which are currently utilized industry transport protocols. The panel identified SOAP as the protocol that can meet the current and future needs of Immunization Information System (IIS) data exchange and that also has the best chance for broad adoption across disparate healthcare systems [12] but HIT vendors are not mandated to use any particular transport protocol for ELR.

It is essential to note that KHIE relies heavily on VPN and SOAP for transport protocols but the SME from CDC did not include VPN as one of the five transport protocol options. KHIE does not know whether its vendors encounter any challenges in using VPN or SOAP though that is what they rely on. Understanding challenges related to securely transporting ELR by vendors was one of the essential goals to KHIE to improve their reporting system and reduce barriers. In addition, the HL7 standardized interface implementation guide contains a lot of information. 
KHIE wants to know whether vendors clearly understand the requirements in the implementation guide or have challenges with some of the vocabularies.

Historically, most laboratories developed their own set of local codes to describe their findings [3]. This has never been a good practice but currently ELR standards specify the use of Logical Observation Identifiers Names and Codes (LOINC) for reporting the type of test, but many laboratories are not yet capable of specifying results using LOINC codes. Even when they do have that capability there are substantial differences between versions of LOINC [3]. It would interest KHIE to understand any challenges vendors encounter when using the HL7 implementation guide. Harmonizing these values is an important and challenging aspect of implementing the ELR [3]. In order to have successful ELR implementation, there are three areas that KHIE needs comprehensive feedback through evaluation and analysis. These are transport protocols, reportable diseases, and semantic interoperability (standardized interface implementation). Currently, KHIE has not explored these areas to find out if vendors have any challenges in meeting the requirements of ELR.

That notwithstanding, KHIE vendors have been working hard to meet the requirements for ELR certification as expected. However, less attention has been paid to understanding the challenges the vendors go through to meet these requirements. Thus understanding vendors' challenges in meeting the requirements for MU and ELR is essential to ensure quality laboratory reporting. Therefore, the purpose of this study was to explore the challenges that HIT vendors face when satisfying the requirements of Meaningful Use and Electronic Laboratory Reporting on reportable diseases to the public health departments in Kentucky.

\subsection{Study Design and Sample}

The study was cross-sectional. Participants were recruited from Kentucky Health Information Exchange HIT vendors. All of the participants were vendors in Kentucky who were involved in Meaningful Use and Electronic Laboratory Reporting. Individuals were selected based on their position as the representative or contact person of their organization. Participants were recruited by email through KHIE vendors' contact list. The list was comprised of 55 representatives of vendors who work with KHIE with their company names, product types, certification year and contacts.

\subsection{Recruitment and Data Collection}

An active recruitment method was used to appeal to the current vendors who qualified for the study. Vendors who had contact details up to the end of the year 2013 or later with KHIE were actively recruited to participate in the study. In all, nine (9) questions were included in the survey.

Out of the 55 vendors who received the survey, 35 (63.64\%) responded to the survey. There was fourteen (14) reminder notices and three emails sent to vendors to increase participation. The reminder notices and emails helped to increase participation. The data was collected from February 2014 through March 2014. Thus, the sample analysis was comprised of 35 KHIE 
vendors' representatives who were authorized by their organizations to share their challenges with KHIE.

The survey questions were carefully designed and discussed with the KHIE subject matter expert (SME), an expert in public health, and another in biomedical and health informatics. The survey focused on three important areas to collect data from the vendors: Transport Protocols (Focus A), Reportable diseases (Focus B) and Semantic interoperability (Standardized Interface Implementation) (Focus C). Online survey software (Survey Monkey) was used to make it easy for vendors to provide responses. Vendors who could not respond to the survey through the Survey Monkey link also received a copy of the questions attached to their email and could email back after completion.

A brief introduction about the study was included as to why they had been contacted to participate in the survey and what the study wanted to achieve. A reminder email was sent to vendors one week after they received the survey link to increase participation.

\subsection{Measures}

Focus A concerned challenges related to securely transporting ELR. Understanding challenges that vendors face regarding transport protocols was one of the essential needs of KHIE for improving their reporting system and reducing barriers. The questions in this section were used to find out which of the transport protocols was difficult to implement and if vendors had preferences regarding which protocol they would adopt. Seven (7) examples of transport protocols were used in the survey. These include; Secure FTP(sFTP), Simple Object Access Protocol (SOAP) web service, ebXML via the Public Health Information Network Messaging System (PHINMS), Direct protocol- Simple Mail Transfer Protocol(SMTP), Secure/Multipurpose Internet Mail Extensions (S/MIME), Hypertext Transfer Protocol Secure (HTTPS POST), Hypertext Transfer Protocol Secure Representational State Transfer (HTTPS REST), Minimum Lower Layer Protocol(MLLP), and VPN such as Point-to-Point Tunneling Protocol (PPTP) and Layer 2 Tunneling Protocol (L2TP). Vendors were asked to select the most challenging transport protocols for ELR and state if they have a preference, which protocol they would prefer for ELR.

Focus B was about Reportable Diseases: The questions in this section were used to explore what is reportable by jurisdiction and difficulties in detecting what is reportable in different jurisdictions. The following two questions were asked: 1) Do you see any variability in what is reportable by jurisdiction? and -2) Is it difficult to detect what is reportable in Jurisdiction A versus in $\mathrm{B}$ ?

The purpose of Focus C was to identify if there are any challenges in HL7 Standard Implementation Guide required by Meaningful Use for 2014 ELR certification. A continued challenge for broader uptake of ELR is the slow rate of adoption of the messaging and vocabulary standards that are intended to make the sharing of information easier and more useful [3]. The HL7 standard implementation guide provides useful guidelines to all users to ensure effective and quality reporting. The Health IT Standards Committee, which advises the National Coordinator for Health IT, recently recommended a minimum necessary set of vocabulary 
standards that will enable interoperable electronic health record data elements [13]. These vocabulary standards specify LOINC for laboratory tests and diagnostic studies as well as many other categories of information [14].

With this in mind, questions were asked to explore whether vendors have any challenges with the HL7 standard implementation guide required by Meaningful Use for 2014 ELR certification, why they find a particular standard challenging, vocabularies that they have the greatest challenge with, and from where they get their vocabulary standard for Public Health ELR.

\section{Data Analysis and Results}

The survey was initially sent to 55 KHIE vendors. Though, KHIE has more than 55 vendors, they did not have updated email addresses for the rest of the vendors at the beginning of the survey.

Of the 55 potential participants, 35 (63.64\%) completed the survey. Respondents had the option to skip questions as desired or opt-out entirely. A simple descriptive analysis was conducted to look at the frequencies and percentages of the responses toward each variable. Cross tabulation and Chi-Square analyses were then conducted on four variables. All analyses were conducted using IBM SPSS Statistics for windows version 22.0.

In all there were nine (9) questions of interest in the survey. The first question was to determine the most challenging transport protocol to implement. Out of the seven (7) transport protocol options, vendors selected VPN (PPTP and L2TP) as the most difficult to implement (31.7\%). See Figure1. A chi-square goodness of fit test was calculated comparing the frequency of occurrences of each value of transport protocol. It was hypothesized that each value would occur an equal number of times. Significant deviation from the hypothesized values was found ( $\mathrm{X}$ $[2](5)=16.94, \mathrm{P}<.05)$. The null hypothesis was rejected.

SFTP was selected as the preferred ELR transport protocol (31.4\%). A chi-square goodness of fit test was calculated comparing the frequency of occurrences of each value of transport protocol. It was hypothesized that each value would occur an equal number of times. Significant deviation from the hypothesized values was found $(\mathrm{X}[2](5)=11.80, \mathrm{P}<.05)$. The null hypothesis was rejected. Again, a Pearson chi-square test of independence was calculated comparing the frequency of transport protocols in question 1 and question 2. It was hypothesized that vendors have challenges with transport protocols and would prefer to use a particular transport protocols if they have a preference. No significant deviation from the hypothesis was found $(X[2](25)=$ $28.42 \mathrm{P}>.05$ 
Figure1: Of the following transport protocols, which do you find is most challenging to implement?

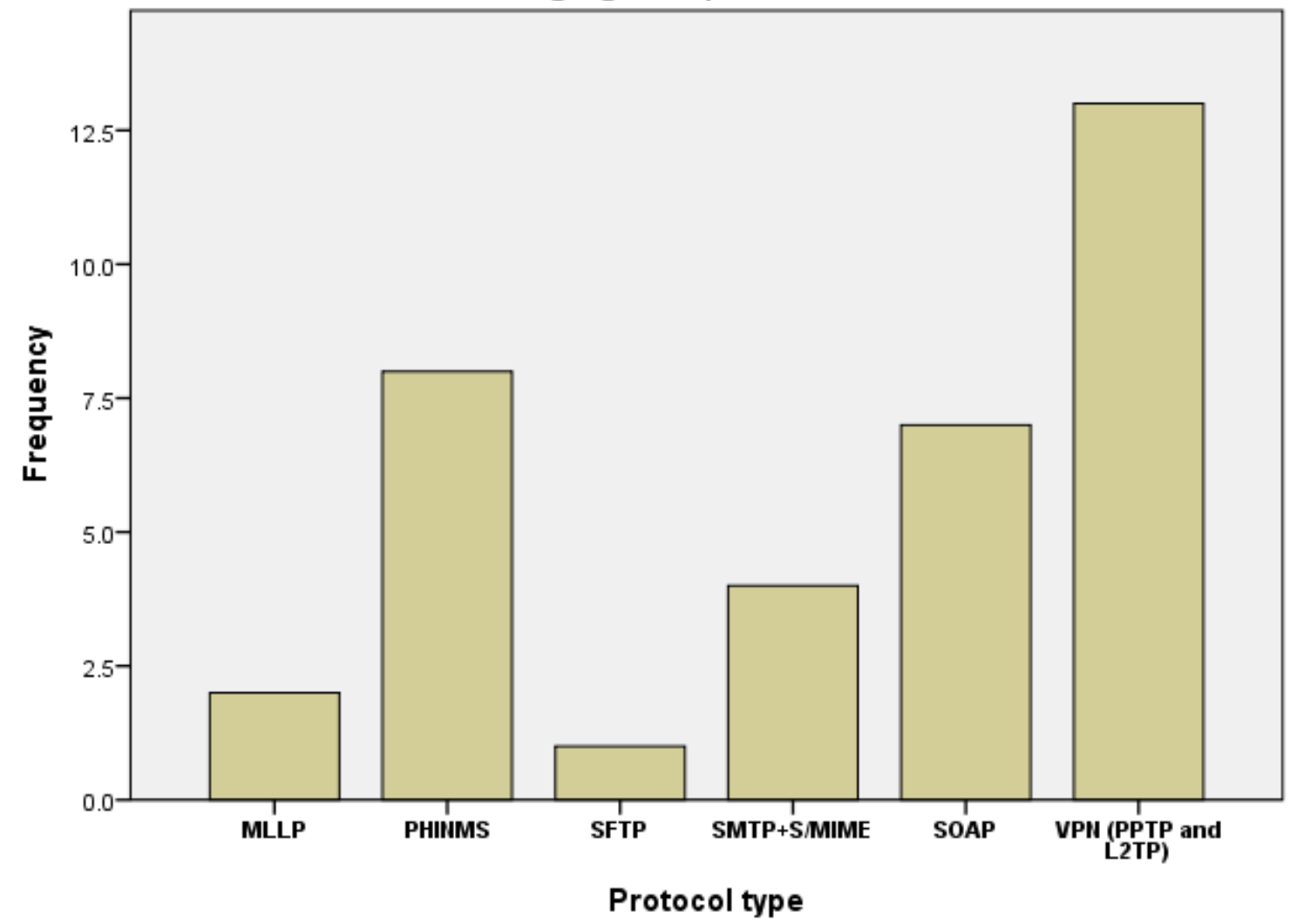

Figure 1: Of the following transport protocols, which do you find is most challenging to implement?

The next focus area was on reportable diseases. Almost 54.3\% of the respondents answered NO to indicate they do not see any jurisdictional differences in reportable diseases while 45.7\% (16 of 35 respondents) answered YES to the effect that they do see variability in what is reportable by different jurisdictions. A chi-square goodness of fit test was calculated comparing the frequency of occurrences of each value of a response. It was hypothesized that each value would occur an equal number of times. No significant deviation from the hypothesized values was found $(\mathrm{X}[2](1)=.28, \mathrm{P}>.05)$. The null hypothesis was retained. See table 1 below for details.

Besides, about $62.9 \%$ (22 of respondents) answered NO to show that it is not difficult to detect what is reportable in different jurisdictions. A chi-square goodness of fit test was calculated comparing the frequency of occurrences of each value of a response. It was hypothesized that each value would occur an equal number of times. No significant deviation from the hypothesized values was found $(\mathrm{X}[2](5)=2.31, \mathrm{P}>.05)$. The null hypothesis was retained. In addition, a Pearson chi-square test of independence was calculated comparing the frequencies of question 3 and 4. It was hypothesized that vendors do not see any variability in what is reportable by jurisdiction and it is not difficult to detect what is reportable in different jurisdictions. No 
significant deviation from the hypothesis was found $(\mathrm{X}[2](1)=2.08 \mathrm{P}>.05$. See Table 1 below for details.

Table 1

\begin{tabular}{|c|c|c|c|}
\hline \multicolumn{4}{|c|}{ 3. Do you see any variability in what is reportable by jurisdiction? } \\
\hline \multirow[b]{2}{*}{ NO } & Frequency & Percent & Chi-Square \\
\hline & 19 & 54.3 & \\
\hline YES & 16 & 45.7 & $(\mathrm{X}[2](1)=.28, \mathrm{P}>.05)$ \\
\hline Total & 35 & 100 & \\
\hline \multicolumn{4}{|c|}{ 4. Is it difficult to detect what is reportable in Jurisdiction A than in B? } \\
\hline & Frequency & Percent & Chi-Square \\
\hline NO & 22 & 62.9 & \\
\hline YES & 13 & 37.1 & $(\mathrm{X}[2](5)=2.31, \mathrm{P}>.05)$ \\
\hline TOTAL & 35 & 100 & \\
\hline
\end{tabular}

Focus $\mathrm{C}$ was on semantic interoperability- standardized interface implementation guide. Most of the respondents, $80 \%$, responded that they do not have any challenges with the HL7 standard implementation guide required by meaningful use for 2014 ELR certification. A chi-square goodness of fit test was calculated comparing the frequency of occurrences of each value of a response. It was hypothesized that each value would occur an equal number of times. Significant deviation from the hypothesized values was found $(\mathrm{X}[2](1)=12.60, \mathrm{P}<.05)$. The null hypothesis was rejected. See table 2 for details.

Table 2

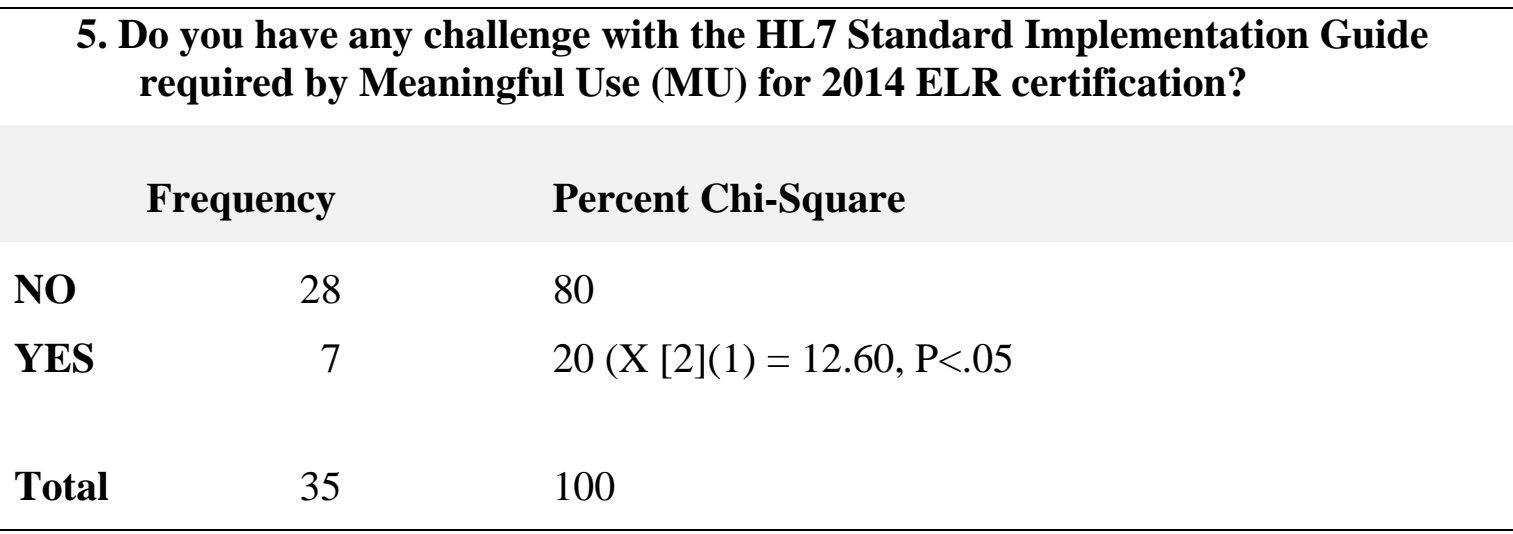

Question 6 and 9 were open-ended questions and responses are summarized under discussion. 
With respect to question number eight (8) which was to identify vocabulary standard vendors have challenge with, about $11(31.4 \%)$ of the respondents selected Unified code for Unit of Measure (UCUM) as vocabulary standard they have difficult challenge while $9(26.7 \%)$ skipped or did not answer the questions. A chi-square goodness of fit test was calculated comparing the frequency of occurrences of each value of a response. It was hypothesized that each value would occur an equal number of times. No significant deviation from the hypothesized values was found $(\mathrm{X}[2](3)=1.0, \mathrm{P}>.05)$. The null hypothesis was retained.

Regarding question number seven (7), which was designed to find out why vendors find particular standard challenging, 34(97.14\%) did not respond.

\subsection{Discussion}

The finding suggests that the most difficult transport protocol to implement for ELR is VPN (PPTP and L2TP). Surprisingly, KHIE relies heavily on VPN for ELR. Does this mean KHIE is increasing the burden on its vendors? Again, if vendors have preference, they would use SFTP for ELR. Could this result be used as a direction towards a selection of SFTP as third transport protocol options for KHIE vendors? The finding is very interesting to note because the 2011 CDC panel of subject matter experts (SMEs) did not select VPN (PPTP and L2TP) as one of the five transport protocol options perhaps due to similar observations that VPN is difficult to implement or it does not support the current industry utilized requirements. SFTP which was selected as the preferred transport protocol was among the five options selected by the CDC panel.

The list of diseases considered reportable varies by state and year. Since states and jurisdictions are sovereign entities, reportable conditions are determined by laws and regulations of each state and jurisdiction [15]. To this end, it is possible that some conditions considered naturally reportable might not be reported in certain jurisdictions. The study found the majority of the HIT vendors in Kentucky do not see any variability in what is reportable by different jurisdiction and that it is not difficult to detect what is reportable from one jurisdiction to another. Reporting completeness of notifiable diseases is highly variable and related to the condition or disease being reported [16].

The finding again stipulates that vendors in general do not have any challenges with the HL7 standard implementation guide required by Meaningful Use ELR certification. However, some vendors stated that they do have challenges with some of the vocabularies used in the HL7 implementation guide. In particular, if a vendor uses vocabularies that are not up to date, it will affect its data quality and create confusion in the reporting system. It has been observed that delays in development or distribution of an updated version of the vocabulary may result in some laboratories temporarily reverting to paper reporting systems or mapping new aggregate concepts to existing but inaccurate terms [15]

The results from the survey show that vendors have greatest challenge with UCUM. Previous studies found out that vast expressive power, concept richness, and flexibility of a post coordinated vocabulary seem better suited to public health surveillance requirements and the diverse information system capabilities of laboratories, but the post coordinated vocabulary may 
require additional guidelines for the composition of appropriate and consistent terms [14]. Vendors' having deep understanding of the vocabulary standard for ELR is critical for quality laboratory reporting.

According to some vendors "HL7 implementation is time consuming and could take a team of three (3) approximately five (5) years to implement HL7 successfully which is too long for any manager to commit resources for implementation”. No specific reason was assigned to why HL7 implementation is time consuming as stated by some respondents. Some vendors also mentioned that new required data fields which are not currently being captured by customers make it difficult to incorporate into workflow.

Another area of interest to KHIE was to determine where vendors get their vocabulary standard from. ELR reporting may be inaccurate if the source does not have an updated vocabulary standard. Vendors revealed the following sources of vocabulary standard: Center for Disease Control and Prevention (CDC), National Library of Medicine, InfoGuard Laboratories Inc, eMDS, Medicapaedia, Centers for Medicare and Medicaid Services (CMS) guidelines, Unified Medical Language System (UMLS), Terminology Services and Regenstrief LOINC Mapping Assistant (RELMA). This finding suggests that vendors get their vocabulary standard from various sources. This is a major concern for KHIE that warrants further investigation.

\subsection{Recommendation}

As part of KHIE aims to reduce barriers and improve the ELR system, the following recommendation should be taken into consideration.

KHIE should recommend a maximum of three (3) transport protocols that have the best chance of adoptions across disparate HIT vendors to move towards the standardization in ELR in Kentucky.

KHIE should investigate why vendors prefer SFTP over their choice (VPN and SOAP)

Further research is needed to explore the difficulties in implementing VPN and SOAP and what can be done to make it less burdensome since KHIE heavily relies on these two transport protocol options. There is also a need to zero in on why some vendors see variability and find it difficult to detect what is reportable in different jurisdictions. Again, there should be follow up studies to find out why HL7 is time consuming to some vendors.

\subsection{Limitations}

Some vendors' representatives did not respond to the survey because some of the questions appeared to be too technical for them to answer. Those who could not respond to the survey questions due to the technicalities involved were either project managers or administrators who lack the technical understanding of the questions. They promised to get resources from their organization to answer the questions but could not do so before the end of the data collection. The duration of the data collection might have been too short for some vendors to respond to the survey. The contact list of KHIE vendors did not comprise all of their vendors which limited the 
number of vendors who could have participated or responded to the survey and possibly altered the outcome of the results. A follow up phone survey could have increased the participation but KHIE did not have vendors' telephone numbers on their contact list.

\section{Conclusion}

The result of the study has demonstrated the importance of understanding vendors' challenges in meeting the requirements for ELR to ensure quality laboratory reporting. The study found that the most difficult transport protocol to implement for ELR is VPN (PPTP and L2TP) and if vendors have preference, they would use SFTP for ELR over KHIE choice of VPN and SOAP. Most of KHIE vendors do not see any variability in what is reportable by different jurisdiction and also it is not difficult for them to detect what is reportable from one jurisdiction versus the other.

Again, vendors in general do not have any challenge with the HL7 standard implementation guide required by Meaningful Use ELR certification. However, some vendors mentioned that HL7 implementation is time consuming and could take a team of three (3) approximately five (5) years to implement HL7 successfully which is too long for any manager to commit resources for implementation.

As part of addressing some of the challenges identified by this study, KHIE should recommend a maximum of three (3) transport protocols that have the best chance of adoptions across disparate HIT vendors to move towards the standardization in ELR in Kentucky.

\section{Acknowledgements}

This research wouldn't have been possible without the blessing and favor from the Almighty God and wonderful support from my family, professors, mentors and friends. I would like to thank those who have helped me during the writing of this paper.

I want to express my heartfelt appreciation to Dr. Martha C. Riddell, Dr. William G. Pfeifle, Dr. Todd R. Johnson from University of Kentucky and Brennan O’Banion from KHIE for their wonderful support throughout the writing process.

\section{GLOSSARY OF TERMS AND PHRASES}

Bioterrorism; According to the U.S. Centers for Disease Control and Prevention a bioterrorism attack is the deliberate release of viruses, bacteria, toxins or other harmful agents used to cause illness or death in people, animals, or plants

Data: Information.

Electronic Laboratory Reporting (ELR): Electronic Laboratory Reporting is the electronic transmission from laboratories to public health of laboratory reports which identify reportable conditions. ELR has many benefits, including improved timeliness, reduction of manual data entry errors, and reports that are more complete (http://www.cdc.gov/ehrmeaningfuluse/elr.html)

e-MDs: This is a developer of healthcare software solutions. The company is headquartered in Austin, Texas and was founded in 1996 by David Winn, MD. The company remains physician-managed, and actively participates in national health information and interoperability efforts 
Health Information Exchange (HIE): The movement of healthcare information electronically across organizations within a region or community. HIE provides the capability to electronically move clinical information between disparate healthcare information systems while maintaining the meaning of the information being exchanged. The goal of HIE is to facilitate access to and retrieval of clinical data to provide safe, timely, efficient, effective, equitable, patient-centered care.

Health Information Technology (HIT): The application of information processing involving both computer hardware and software that deals with the storage, retrieval, sharing, and use of health care information, data, and knowledge for communication and decision making.

HL7 (Health Level Seven): One of several accredited standards (specifications or protocols) established by ANSI (American National Standards Institute) for clinical and administrative data. Systems which are HL7 'compliant' improve the ability for interoperability and exchange of electronic data.

Hypertext Transfer Protocol (HTTP): A language protocol used in communication among Web sites.

Hypertext Transfer Protocol Secure (HTTPS): This is a communications protocol for secure communication over a computer network, with especially wide deployment on the Internet (Wikipedia)

Interoperability - Compatibility: The ability of software and hardware on multiple pieces of equipment made by different companies or manufacturers to communicate and work together

National Institute of Standards and Technology (NIST): Founded in 1901, NIST is a non-regulatory federal agency within the U.S. Commerce Department's Technology Administration, promoting U.S. innovation and industrial competitiveness by advancing measurement science, standards, and technology.

Logical Observation Identifiers Names and Codes (LOINC): This is a database and universal standard for identifying medical laboratory observations. It was developed and is maintained by the Regenstrief Institute, a US non-profit medical research organization, in 1994. LOINC was created in response to the demand for an electronic database for clinical care and management and is publicly available at no cost (Wikipedia)

Meaningful Use (MU): The Medicare and Medicaid EHR Incentive Programs which provide financial incentives for the "meaningful use" of certified EHR technology to improve patient care.

Minimal Lower Layer Protocol (MLLP): Defines the leading and trailing delimiters for an HL7 message. These delimiters help the receiving application to determine the start and end of an HL7 message that uses Internet Protocol network as transport

Multipurpose Internet Mail Extensions (MIME): This is an Internet standard that extends the format of email to support: Text in character sets other than ASCII Non-text attachments Message bodies with multiple parts (Wikipedia)

National Library of Medicine (NLM): The world's largest biomedical library, NLM maintains and makes available a vast print collection and produces electronic information resources on a wide range of topics that are searched billions of times each year by millions of people around the globe. It also supports and conducts research, development, and training in biomedical informatics and health information technology

Office of the National Coordinator (ONC): Is a government agency (part of HHS) that oversees and encourages the development of a national, interoperable (compatible) health information technology system to improve the quality and efficiency of health care.

Public Health Information Network Messaging System (PHINMS): The Centers for Disease Control and Prevention (CDC)'s Public Health Information Network (PHIN) is a national initiative to increase the capacity of public health agencies to electronically exchange data and information across organizations and jurisdictions (e.g., clinical care to public health, public health to public health and public health to other federal agencies).

Reportable diseases: Reportable diseases are diseases considered to be of great public health importance. Local, state, and national agencies (for example, county and state health departments or the U.S. Centers for Disease Control and Prevention) require that these diseases be reported when they are diagnosed by doctors or laboratories (http://www.nlm.nih.gov/medlineplus/ency/article/001929.htm) 
Secure File Transport Protocol (SFTP). Secure File Transfer Protocol is an interactive file transfer program which performs high speed file transfer operations over an encrypted secure shell (ssh) transport.

Simple Mail Transfer Protocol (SMTP): Simple Mail Transfer Protocol is an Internet standard for electronic mail transmission. (Wikipedia)

Simple Object Access Protocol (SOAP). Originally defined as Simple Object Access Protocol, is a protocol specification for exchanging structured information in the implementation of Web Services in computer networks (Wikipedia)

Subject matter expert, or SME A "person with bona fide expert knowledge about what it takes to do a particular job. First-level supervisors are normally good SMEs. "(Delegated Examining Operations Handbook

Survey Monkey: Survey Monkey is a web survey development cloud based company, founded in 1999 by Ryan Finley (Wikipedia)

Terminology Services and Regenstrief LOINC Mapping Assistant (RELMA): The Regenstrief Institute provides a Windows-based mapping utility called the Regenstrief LOINC Mapping Assistant (RELMA) to facilitate searches through the LOINC database and to assist efforts to map local codes to LOINC codes (http://loinc.org/relma)

Transport Protocols. In computer networking, a transport layer provides end-to-end or host-to-host communication services for applications within a layered architecture of network components and protocols (Wikipedia)

Unified Code for Unit of Measure (UCUM): The Unified Code for Units of Measure is a code system intended to include all units of measures being contemporarily used in international science, engineering, and business. The purpose is to facilitate unambiguous electronic communication of quantities together with their units. (http://unitsofmeasure.org/trac/)

Unified Medical Language System (UMLS): The UMLS, or Unified Medical Language System, is a set of files and software that brings together many health and biomedical vocabularies and standards to enable interoperability between computer systems

Virtual Private Network (VPN): A network that uses public connections, such as the Internet, to link users but relies on encryption and other security measures to ensure that only authorized users can access the network

\section{Conflicts of Interest}

None declared.

\section{References}

1. Centers for Disease Control and Prevention. Preventing Emerging Infectious Diseases: A Strategy for the 21st Century. Atlanta, Ga: US Dept of Health and Human Services; 1998.

2. Panackal AA, M'ikanatha NM, Tsui F-C, et al. 2002. Automatic electronic laboratory-based reporting of notifiable infectious diseases at a large health system. Emerg Infect Dis. 8, 685-91. PubMed https://doi.org/10.3201/eid0807.010493

3. Rose D. The value of Electronic Laboratory Reporting for Public Health: Scientific Technologies Corporation: White Paper, 2010

4. New Jersey Department of Health and Senior Services: Electronic Laboratory Reporting Technical Manual. May 2007 
5. Medicare \& Medicaid. EHR Incentive Program. Meaningful Use. Stage 1 Requirements Overview. 2010 (http://www.cms.gov/EHRIncentivePrograms/)

6. Nguyen TQ, Thorpe L, Makki HA, Mostashari F. 2007. Benefits and barriers to electronic laboratory results reporting for notifiable diseases: the New York City Department of Health and Mental Hygiene experience [Am J Public Health]. Am J Public Health. 97(Suppl 1), S142-45. PubMed https://doi.org/10.2105/AJPH.2006.098996

7. Klaucke D, Buehler J, Thacker S, Parrish R, Trowbride F, et al. 1988. Guidelines for evaluating surveillance systems. MMWR Morb Mortal Wkly Rep. 37, 1-15. PubMed

8. Effler P, Ching-Lee M, Bogard A, Ieong M, Nekomoto T, et al. 1999. Statewide system of electronic notifiable disease reporting from clinical laboratories: comparing automated reporting with conventional methods. JAMA. 282, 1845-50. PubMed https://doi.org/10.1001/jama.282.19.1845

9. US Centers for Disease Control and Prevention: Automated detection and reporting of notifiable diseases using electronic medical records versus passive surveillance--Massachusetts, June 2006-July 2007: MMWR: Morbidity \& Mortality Weekly Report (MMWR MORB MORTAL WKLY REP), 2008 Apr 11; 57 (14): 373-6. (10 ref)

10. Lee L, Whitcomb K, Galbreth M, Patterson D. 2010. A Strong State Role in HIE: Lessons from the South Carolina Health Information Exchange. J AHIMA. 81(6), 46-50. PubMed

11. Noam H. Arzt Slide c/o Office of the National Coordinator (Meaningful Use Public Health Forum \& CDC Nationwide Call March 21, 2013:HLN Consulting, LLC)

12. Centers for Disease Control and Prevention - National Center for Immunization and Respiratory Diseases, \& EHR-IIS Interoperability Expert Panel Project. 2011.

(http://www.cdc.gov/vaccines/programs/iis/interop-proj/ehr.html.)

13. Clinical quality measures workgroup and vocabulary task force (Health Information Technology Standards Committee, Washington, DC). Letter to: Dr. Farzad Mostashari, National Coordinator for Health Information; 2011 Aug 17. <http://healthit.hhs.gov/portal/server.pt/gateway/PTARGS_0_12811_955546_0_0_18/HITS C_CQMWG_VTF_Transmit_090911.pdf>

14. Abhyankar S, Demner-Fushman D, McDonald CJ. 2012. Standardizing clinical laboratory data for secondary use. J Biomed Inform. 45(4), 642-50. PubMed https://doi.org/10.1016/j.jbi.2012.04.012

15. Mark D. 1999. White, Linda M Kolar, Steven J Steindel: Evaluation of Vocabularies for Electronic Laboratory Reporting to Public Health Agencies. J Am Med Inform Assoc. 6, 185-94. doi:https://doi.org/10.1136/jamia.1999.0060185. PubMed

16. Center for Disease Control and Prevention. Morbidity and Mortality Weekly Report (MMWR): Summary of Notifiable Diseases. Available at: www.cdc.gov/mmwr/mmwr_nd. Accessed March 26, 2014. 
17. Revere D; Stevens KC: Accelerating Public Health Situational Awareness through Health Information Exchanges: An Annotated Bibliography: Online Journal Of Public Health Informatics [Online J Public Health Inform]. 2010. Vol. 2 (2). Date of Electronic Publication. 2010(Oct), 29. 